\title{
REVISIONES
}

\section{Educación ambiental y cultura evaluativa. Algunas reflexiones para la construcción de eco-consciencias}

\author{
Environmental Education and evaluative practice. \\ Some thoughts for the construction of eco-consciousness
}

David Herrera Araya, ${ }^{a}$ Daniel Ríos Muñoz ${ }^{b}$

aDepartamento de Educación, Universidad de Santiago de Chile Telf.: (56) 982416252. Correo electrónico: david.herrera@usach.cl

bTelf.: (56) 227184651. Correo electrónico: daniel.rios@usach.cl

\section{RESUMEN}

Este artículo tiene como propósito reflexionar sobre las relaciones e interacciones entre la Educación Ambiental (EAM) y la práctica evaluativa. La intención es elaborar una propuesta evaluativa que aporte a la construcción de sujetos eco-conscientes y eco-responsables. La premisa subyacente plantea que la escuela es actualmente un espacio privilegiado para la generación e implementación de prácticas educativas novedosas que contribuyan a la protección del medio ambiente. El fundamento central sostiene que el desarrollo de una práctica evaluativa orientada a la formación de eco-consciencias en el aula permite avanzar y consolidar los aprendizajes para la vida.

Palabras clave: Educación Ambiental (EAM), cultura evaluativa, eco-consciencia.

\begin{abstract}
The purpose of this article is to reflect on the relationships and interactions between Environmental Education (EE) and the evaluative practice. The principal aim is to develop an evaluative proposal that contributes to the education of eco-conscious and eco-responsible people. The underlying premise states that the school is, nowadays, a privileged place to generate and introduce innovative educational practices that contribute to protect the environment. The main basis of this article is that the development of an evaluative practice of ecoconsciousness-oriented training in the classroom allows to improve and strengthen learning for life.
\end{abstract}

Key words: Environmental Education (EE), evaluative practice, eco-consciousness. 


\section{INTRODUCCIÓN}

Las experiencias en Educación Ambiental (EAM) al interior de las escuelas han ido adquiriendo progresivamente una mayor relevancia. Desde la creación en Chile del Ministerio del Medioambiente, en el 2010, el país se ha esforzado por establecer medidas y lineamientos institucionales y educativos para fomentar el desarrollo sustentable, con la finalidad de mejorar la calidad de vida de las personas.

Podemos destacar los avances en esta línea del trabajo gubernamental a partir de los tres ejes definidos por el Estado de Chile como prioritarios en el área medioambiental: la Gestión Ambiental ligada a la Certificación Ambiental Municipal y al Programa Barrios Sustentables; la Educación Ambiental que incluye el Programa de Certificación de escuelas y el desarrollo de planes y programas para la capacitación en liderazgo ambiental y eco-responsable; y, finalmente, el Fondo de Protección Ambiental para la ciudadanía (Ministerio del Medioambiente, 2015). Todos estos planes se agregan al desafío de las energías renovables no convencionales, a la nueva normativa de impacto ambiental, entre otros. Con estas iniciativas, las políticas públicas han demostrado una preocupación por la formación de una cultura ambiental sustentable al interior de la ciudadanía en general.

Los esfuerzos actuales al interior de las escuelas radican en la incorporación de planes de mejoramiento educativo vinculados a la transformación de los espacios comunes. Por ejemplo, el impulso de zonas para el reciclaje, la creación de talleres sobre el cuidado del medio ambiente y las alianzas con diferentes organizaciones privadas y públicas para el trabajo sobre la importancia de la EAM (Ministerio del Medio Ambiente, 2016). Las experiencias existentes a nivel nacional son reforzadas desde el Programa de Certificación Ambiental Municipal y con una serie de concursos públicos para acceder a fondos que desarrollen políticas de desarrollo sustentable.

En lo que respecta a la EAM, el Estado ha desarrollado principios educativos generales, basados en los fundamentos internacionales contenidos en el documento "Decenio de las Naciones Unidas para el Desarrollo Sostenible" (Delors, 2006). En concordancia con estos principios, el objetivo general de la EAM definida por Chile es

Formar personas y ciudadanos capaces de asumir individual y colectivamente la responsabilidad de crear y disfrutar de una sociedad sustentable y contribuir al fortalecimiento de procesos educativos que permitan instalar y desarrollar valores, conceptos, habilidades, competencias y actitudes en la ciudadanía en su conjunto. (Ministerio del Medioambiente, 2010, p. 12).

Sin embargo, este objetivo de política nacional ha sido incorporado tímidamente en las bases curriculares como una dimensión más en el desarrollo transversal asociado a la temática de la persona y su entorno, a partir de métodos y actividades aisladas de los niveles de educación básica y media sin cohesión interna y coherencia entre las asignaturas. Sin embargo, existen experiencias impulsadas por el Ministerio del Medioambiente vía proyectos y fondos concursables para incentivar la implementación de talleres en las escuelas básicas (Ministerio del Medioambiente, 2015). Estas experiencias alentadoras en lo curricular y pedagógico no han incluido sistemáticamente el desarrollo de una cultura y práctica evaluativa para la construcción de eco-consciencias que, por definición, es un acto individual y colectivo, $\mathrm{y}$, por lo tanto, se transforma en una responsabilidad socioambiental. 
La persistencia de este desafío educativo representa una oportunidad para generar y construir aportes y así fomentar el desarrollo de una práctica pedagógica y evaluativa a la altura del desafío de la EAM que nos impone el deber moral y ético para colaborar en la protección del medioambiente y el desarrollo sustentable del país.

Primero, presentamos algunas consideraciones conceptuales sobre la EAM, para luego establecer los elementos esenciales sobre la evaluación para la EAM, que incluye principios de metodología evaluativa y consideraciones prácticas a modo de aporte para la formación de una cultura educativa medioambiental. Un tercer acápite referido a la construcción de una propuesta que considere la práctica y cultura evaluativa en EAM y, finalmente, algunas reflexiones sistematizadas para abrir nuevas interrogantes y posibles orientaciones educativas sobre el tema trabajado.

\section{CONSIDERACIONES CONCEPTUALES DE LA EDUCACIÓN AMBIENTAL}

La Educación Ambiental se ha convertido en una temática central para enfrentar la crisis medioambiental actual. Para lograr un cambio efectivo sobre los comportamientos individuales y sociales de los sujetos, la literatura reciente (Álvarez \& Vega, 2009; Cantú, 2014; Gutiérrez \& Pozo, 2006; Novo, 2009; Sandoval, 2012) destaca la necesidad de intervenir los espacios educativos con metodologías, estrategias, diseños evaluativos y didácticas específicas para desarrollar competencias y acciones proambientales (Molano, 2012; Sauvé, 2004). De esta manera, los esfuerzos por implementar una política educativa preocupada por reemplazar la relación entre sociedad y medioambiente son fundamentales para preservar no solo a la naturaleza y sus recursos económicos, sino también para responder al desafío de asegurar la propia existencia humana (Stubbs \& Schapper, 2011).

Como ha sido manifestado por la UNESCO en la Conferencia de Dakar (2000), la educación "es un derecho humano fundamental, un factor decisivo del desarrollo sostenible" (UNESCO, 2005, p. 3), y por ende se debe cambiar la relación con la naturaleza para impulsar el desarrollo sustentable o ecoamigable, convirtiéndose en un derecho y deber inmediato para asegurar a las generaciones presentes y futuras (UNESCO, 1972, 2000, 2005). Es aquí donde la escuela es, a juicio de los especialistas (Freitas, 2006; Novo, 2006; Sauvé, 2000; Vega \& Álvarez, 2005), el espacio privilegiado para transformar las pautas socioculturales de los sujetos y así formar una eco-consciencia y una eco-educación Gutiérrez \& Pozo, 2006). Por lo tanto, no solo es urgente diseñar propuestas curriculares sobre cómo educar acciones ambientales, también es indispensable implementar nuevas "arquitecturas" sobre la práctica docente para su integración a marcos evaluativos asociados a la EAM (Edwards et al., 2010; Heikkinen, 2011), metodologías y culturas evaluativas de enseñanza-aprendizaje en el aula (Molano, 2012; Sandoval, 2012; Ruíz, Barraza \& Ceja, 2009) que involucren a los formadores (Sauvé, 2004). Es decir, debe existir una relación dialéctica entre la teoría y la práctica, pero también entre el diseño curricular y las prácticas pedagógicas que conduzca a la formación de ciudadanos eco-responsables.

Considerando estas premisas, el debate de la temática se ha centrado en qué entendemos por EAM o Educación para el Desarrollo Sustentable (EDS). Gran parte de la literatura mencionada divide los marcos teóricos de la EAM en dos corrientes (Álvarez \& Vega, 2009; Gutiérrez \& Pozo, 2006; Sauvé, 2005; Vega et al., 2007). Aquellas que definen el rol de la EAM desde principios valóricos, pero sin establecer una articulación práctica entre el 
diseño teórico con el pedagógico-práctico y, por otro lado, modelos que integran la teoría de la EAM como fundamentos para diseñar marcos didácticos y estrategias de acción en el espacio educativo.

La EAM ha desarrollado marcos curriculares y educativos tendientes a la redefinición de la cultura ambiental y, por ende, de la educación ambiental. Según Meira y Caride (2006) no existe una cultura ambiental, ya que toda acción derivada de la sociedad implica un impacto sobre el entorno. Esto significa que es contradictorio sostener un desarrollo sustentable sin manifestar las acciones transformadores en las cuales los sujetos se hacen responsables de reinterpretar su relación con el medio ambiente. Por esta razón, la EAM debe ser una práctica social integradora, que se esfuerza por satisfacer las necesidades individuales y colectivas desde principios como la solidaridad, reivindicando un espacio propio desde los análisis y discusiones realizadas en el aula, pero también en la comunidad.

El modelo socio-constructivista ofrece una oportunidad interesante para integrar y complementar las prácticas educativas, pedagógicas y evaluativas en cuanto centra sus propuestas en la construcción de saberes integrales contextualizados y prácticos a partir de una interacción dialógica y dialéctica entre estudiante y docente — sujeto a sujeto- para un aprendizaje común y con la capacidad de transformar las prácticas cotidianas fuera del aula (Clará \& Maurí, 2010). También propone una reinterpretación de la EAM a partir de una orientación de construcción de aprendizajes compartidos en interacción con la necesidad de instalar al interior de la escuela el desafío de un desarrollo sustentable que va más allá de los principios básicos de la economía (Cantú, 2014). Es decir, debe responder a cinco funciones básicas para romper con la lógica de que el medioambiente es sencillamente un espacio productivo para el progreso industrial-tecnológico.

Para que la EAM se transforme en nuevo eje de cambio al interior de una sociedad sustentable debe

(1) liberar y dignificar a las personas, al edificar valores más profundos que se vinculen con la conciencia; (2) impulsar cambios en la sociedad para que esta se constituya más íntegra y justa; (3) perfeccionar las potencialidades de todas las personas; (4) aleccionar a los individuos para el trabajo y (5) desarrollar entre las colectividades humanas la sociabilidad y el respeto por la diversidad de culturas. (Cantú, 2008, p. 42).

Como señalan Vilches y Gil (2015), se convierte en una nueva revolución científica en cuanto la EAM posibilita hacer una análisis crítico sobre la insostenibilidad de los recursos naturales, las tasas de contaminación, los comportamientos y acciones individuales y el manejo del progreso científico-tecnológico desligado de la protección del medio ambiente. Por lo tanto la EAM debe ser ecocéntrica (Novo, 2009), es decir, la noción de ser humano eco-dependiente influenciado por su entorno como parte de su propia identidad. Así, los principios de la EAM se establecen como un nuevo modelo de desarrollo educativo que busca reemplazar las lógicas de interacción ser humano-medio ambiente en pro de nuevas acciones frente al desafío de recomponer la armonía de dicha relación.

De esta manera, el principio de la EAM es dialéctico e interdisciplinario (Gutiérrez \& Pozo, 2006), ya que busca integrar los modelos duales de desarrollo social y económico a partir de la incorporación de una eco-educación que ayude a la construcción de una ecoconsciencia. Considerando los principios de Vilches y Gil (2015), Cantú (2014) y Novo (2009), la eco-consciencia es comprendida como una construcción dialógica e integral de 
saberes y prácticas educativas y cotidianas que apuntan a transformar nuestra relación con el medioambiente, en cuanto a su comprensión y mantención para el desarrollo de una mejor calidad de vida. Así, una eco-educación es aquella que permite orientar transversalmente y en forma crítica esta práctica formativa fundamentada en una EAM ecocéntrica.

Según los argumentos esbozados, la EAM debe ser entendida como una acción dialéctica transformadora desde lo curricular y la práctica pedagógica, donde los espacios educativos sean centros de nuevas prácticas individuales y sociales que apunten al desarrollo de saberes, habilidades, valores y actitudes medioambientales. Es así como, desde la construcción de una nueva racionalidad educativa (Molano, 2012), la EAM debe incorporar una construcción desde la crítica sobre las acciones o metacontingencias, entendidas como espacios de intercambio de experiencias y desafíos comunes para cambiar prácticas culturales y valores arraigados socialmente desde la reflexión y la práctica (Sandoval, 2012; Zambrano, Castillo \& Sánchez, 2011). Es en estos espacios de intercambio educativo donde debe ser integrada la evaluación como parte de una cultura holística que coopere en la construcción de sujetos eco-conscientes como una forma de orientar una formación que transcienda el aula.

En este sentido, la dificultad para impulsar una política nacional y local sobre la EAM, radica en la coordinación y cooperación requerida en el nivel institucional —currículum-, la práctica pedagógica y el rol del conjunto social. Tal como plantean Vega et al. (2007), la solución radica en el diseño mismo del modelo didáctico, que debe incorporar una retroalimentación en las estructuras educativas, considerando una integración de principios teórico-prácticos que ha denominado sistémico, de la complejidad y la glocalidad. Según los autores, esto permite responder a las necesidades de integración, relación, retroalimentación de perspectivas, y favorecer el desarrollo de acciones colaborativas para abordar una solución holística del problema ambiental.

\section{LA RELACIÓN ENTRE LA CULTURA EVALUATIVA Y LA PRÁCTICA DOCENTE EN LA EAM}

La construcción de una cultura evaluativa desde la práctica se convierte en un desafío primordial para lograr un cambio en las lógicas existentes sobre la mejora continua de los aprendizajes y la formación de eco-consciencias en el aula. El núcleo de esta lógica se basa en una vasta red de experiencias y concepciones que insertan al docente como un pilar fundamental para reflexionar "en" y "sobre" su visión y praxis educativa (Clará \& Mauri, 2010). Planteado de esta forma, la cultura evaluativa es crucial para impulsar la resignificación al interior de los espacios evaluativos, como una instancia constructiva y democrática a partir de la interacción colectiva como el soporte para la generación de desafíos diversos, pero comunes. Tal como es señalado por Perassi (2010), la evaluación construye y constituye un sujeto de estudio y, por tanto, las interacciones que ocurran al interior de estas dinámicas impactan directamente en los procesos de mejoramiento del aprendizaje.

La relación entre cultura y práctica evaluativa nos lleva a un problema de carácter epistemológico. En los últimos treinta años se ha visto a la evaluación como un campo capaz de contribuir constantemente al desarrollo educativo, interviniendo desde la creación de criterios e indicadores cuantitativos y cualitativos como marcos teóricos para avanzar en el progreso de la calidad global del sistema de enseñanza (Ríos, s/f). Sin embargo, 
surgen complejidades desde la definición misma de "calidad" y su dependencia intrínseca de ciertas valoraciones objetivas y subjetivas que cambian desde la construcción histórica del concepto y, por ende, cargada de percepciones individuales y colectivas. Este problema se asocia al de la práctica docente. Sin querer ampliar el tema propio de la praxis, la transformación y acción docente en el aula evidencia a la evaluación como una pieza anexa al proceso mismo del aprendizaje.

Las experiencias han señalado todo lo contrario. Cuando existe la capacidad del docente para incorporar en su diseño de clase la evaluación en los diferentes momentos y tiempos del aprendizaje, se logran mejores resultados en los procesos de construcción del conocimiento, competencias e, incluso, en desempeños y resultados de carácter más cuantitativos (Moreno, 2010; Rueda, 2006; Salazar, 2010). Esto obliga al docente a incluir en su quehacer cotidiano la experiencia individual y colectiva como sustratos dialécticos para mejorar las prácticas y el sentido de la enseñanza-aprendizaje, considerando las necesidades y el contexto educativo de la escuela y el aula (Rueda, 2004). Por lo tanto, una cultura evaluativa que coloca a la evaluación como un mediador al servicio del estudiante, en cuanto a propósito y fines —en su sentido último-, puede provocar cambios al interior de la realidad escolar (Ravela, 2006).

Es en este espacio donde se articula la EAM y la cultura evaluativa. Como ha sido planteado por González Muñoz (1996), la EAM propone un nuevo entendimiento entre las relaciones del ser humano con el medioambiente, manifestado en la necesidad de establecer nuevas lógicas educativas que cambien las actitudes, valores y consciencias de los sujetos. Esto incluye una nueva visión pedagógica que sea potenciada con experiencias educativas innovadoras a partir de la construcción de un marco educativo distinto. Dicho marco articula la EAM como un tema transversal en las asignaturas, principios éticos, innovación conceptual y metodológica, donde se diseñe una capacidad de evaluación en el proceso educativo.

Estas premisas implicarían observar a la evaluación para la EAM como un mecanismo para la motivación (Moreno, 2010) en virtud del cambio de actitudes y comportamientos sobre el medioambiente. Dicha necesidad requiere una constante autoevaluación de la práctica docente para apreciar la dinámica evaluativa desde un punto de vista multidimensional (Santos Guerra, 1993), incorporando programas educativos medioambientales a partir de la resolución de problemas como eje evaluativo (Zambrano, Castillo \& Sánchez, 2011). Para revelar esas interacciones se debe considerar el espacio evaluativo para que tanto docentes como estudiantes logren establecer lineamientos y esfuerzos comunes desde la cooperación para clarificar las metas que se están estipulando, y así visualizar la evaluación como un espacio de reflexión crítica en pro de la construcción de nuevas conciencias ambientales. Esto nos llevaría a la racionalidad misma del docente, con la finalidad de promover diálogos teóricos y prácticos para incentivar la toma de decisiones, y con ello potenciar la creación de una nueva mirada sobre el desarrollo educativo de la EAM.

La cultura evaluativa debe establecer una familiarización efectiva de los problemas. Vistos como una oportunidad de mejora para integrar las nociones de un proceso democrático real y no meramente burocrático. Esto significa, tal como ha sido planteado por la literatura de las racionalidades curriculares (Pascual, 1998), ir avanzando en posturas dialécticas, donde la realidad educativa se construye bajo actos de negociación y acuerdo.

Esta realidad subjetiva del espacio educativo tendría como efecto, según lo que hemos planteado, una revisión de la cultura evaluativa desde la práctica como fenómeno 
de proceso, en tanto productor de valores y dinámica transformativa (Mateo \& Martínez, 2008). Esto representaría un espacio favorable para la construcción democrática de una cultura evaluativa ambiental, en el sentido que permite vincular y hacer partícipes a los sujetos, a modo de espacio transversal de desarrollo pedagógico (Velásquez, 2009), logrando compromisos mayores en pro de un cambio de actitud sobre el cuidado y protección del medio ambiente.

Por esta razón, se propone apreciar la cultura evaluativa como un constructo complejo y multidimensional que entrega la posibilidad de resignificar el conocimiento y la consciencia del sujeto, desde una dialéctica integrada entre la praxis, el aprendizaje y la realidad educativa. Es el espacio privilegiado en donde confluyen el presente y futuro de la educación y calidad en cuanto construcción sociohistórica, convirtiéndolo en una arquitectura de la justicia social (Escudero, 2003; Gimeno, 2001) y, por lo tanto, como un componente fundamental en el desarrollo de las eco-consciencias.

\section{ELEMENTOS INTEGRALES PARA UNA EVALUACIÓN DE LA EDUCACIÓN AMBIENTAL EN EL AULA}

Para resignificar el proceso de construcción de eco-consciencias en el aula, es necesario referirse a qué perspectiva evaluativa se debe integrar el proceso formativo. A partir de esta premisa, existen dos oportunidades interesantes para ser aplicadas a la EAM. Siguiendo a Ahumada (2001, 2005) y Díaz-Barriga y Barroso (2014), la evaluación auténtica ofrece una visión de proceso significativa que evidencia el desarrollo efectivo de los sujetos en relación a los aprendizajes, habilidades y desempeños logrados mediante la interacción y construcción de actividades relacionadas con tareas específicas. Esto significa que la evaluación auténtica permite diseñar un artefacto esencial para la implementación de una práctica evaluativa orientada al aula y en constante retroalimentación sobre los logros de los procesos educativos en la formación de eco-consciencias.

Desde una perspectiva socio-constructivista (Coll, 2001; Díaz-Barriga, 2014; Perrenoud, 2004), el aprendizaje se basa en una acción activa y consciente, desde la experiencia del sujeto para dar sentido a la construcción de significados y contenidos que son aprendidos en el aula. Este principio, vinculante y sustancial a la concepción evaluativa que estamos presentando, intenciona la acción evaluativa como un componente fundamental para retroalimentar las experiencias educativas en el que participan los sujetos.

En nuestro caso, el interés por la formación de eco-consciencias apunta a la transformación de lo actitudinal — la dimensión del ser- que se enlazaría positivamente con estas miradas sobre el aprendizaje. Aprender es un acto intelectivo, social, afectivo en interacción con las prácticas colectivas/subjetivas que suceden al interior del aula, determinado por lenguajes, constructos y formas educativas que dan cuerpo a las acciones pedagógicas en una intención-acción de carácter reflexiva. Esta mirada es la que ofrece una oportunidad para evaluar la EAM desde un triple enfoque: colaborativo, crítico y experiencial (Chávez \& Larraguibel, 2015; Medir, Heras \& Magin, 2013; Sauvé, 2001; Vallejo \& Molina, 2014).

Para integrar estas nociones, se propone un diseño de implementación que hemos llamado Evaluación para la Formación de Eco-consciencias en el Aula (EFEA) basado en los principios socio-constructivistas para la integración con la EAM desde una perspectiva 
formadora de sujetos eco-conscientes. Al respecto, es importante incluir los siguientes elementos complementarios y/o aclaratorios desde lo educativo y evaluativo para que sean considerados en sus diferentes etapas de formulación (Sauvé, 2003; Vega et al., 2007).

Debe sustentarse en un enfoque experiencial, desde la acción educativa ambiental cotidiana. Su diseño contempla metodologías y estrategias de resolución de problemas, análisis de casos y de proyectos. Esto permite ampliar las dimensiones del instrumento de evaluación para potenciar los aprendizajes, desempeños y/o competencias deseadas. Para transformarse en un diseño experiencial, debe articularse desde un enfoque crítico, donde los criterios evaluativos incorporan las dimensiones de desarrollo socioeconómico y socioambiental, vinculado a la implementación de la actividad en el aula para la construcción del pensamiento crítico.

Para profundizar la dinámica crítica de la EAM, el diseño promueve el trabajo colaborativo. El desafío de la evaluación debe ser el de acompañar las etapas del trabajo que incluyan dimensiones de autoevaluación — también de coevaluación y heteroevaluacióny de carácter inicial, procesual y de resultado. Aquí también subyacen las posibilidades de una evaluación auténtica (Vallejo \& Molina, 2014), para integrar la estructura evaluativa, los grados de complejidad del aprendizaje, los recursos de las actividades y criterios basados en indicadores múltiples del aprendizaje. Por lo tanto, su enfoque es práctico e interdisciplinario. La formación de eco-consciencias queda sujeta a las necesidades del entorno inmediato, a los desafíos curriculares y, sobre todo, a la relación del estudiante con su medio. Por ello, el carácter dialógico de los saberes que posibilita una mirada holística de la evaluación para realizar asociaciones y relaciones en los resultados del aprendizaje que transcienden al aula. Finalmente, se orienta hacia la formación integral. En los últimos veinte años la educación formal ha insistido en el desarrollo de habilidades cognitivas, prácticas y del "ser" para la construcción de sujetos responsables de sus acciones. Bajo esta premisa, la EAM se convierte en una posibilidad real para introducir cambios en la forma de vida actual, con impactos al interior del tejido social y también en la generación de nuevos niveles de conciencia, es decir, una capacitación para la acción (Álvarez \& Vega, 2009). 
Fig. 1. Estructura de la Evaluación para la Formación de Eco-Consciencias (EFEA)

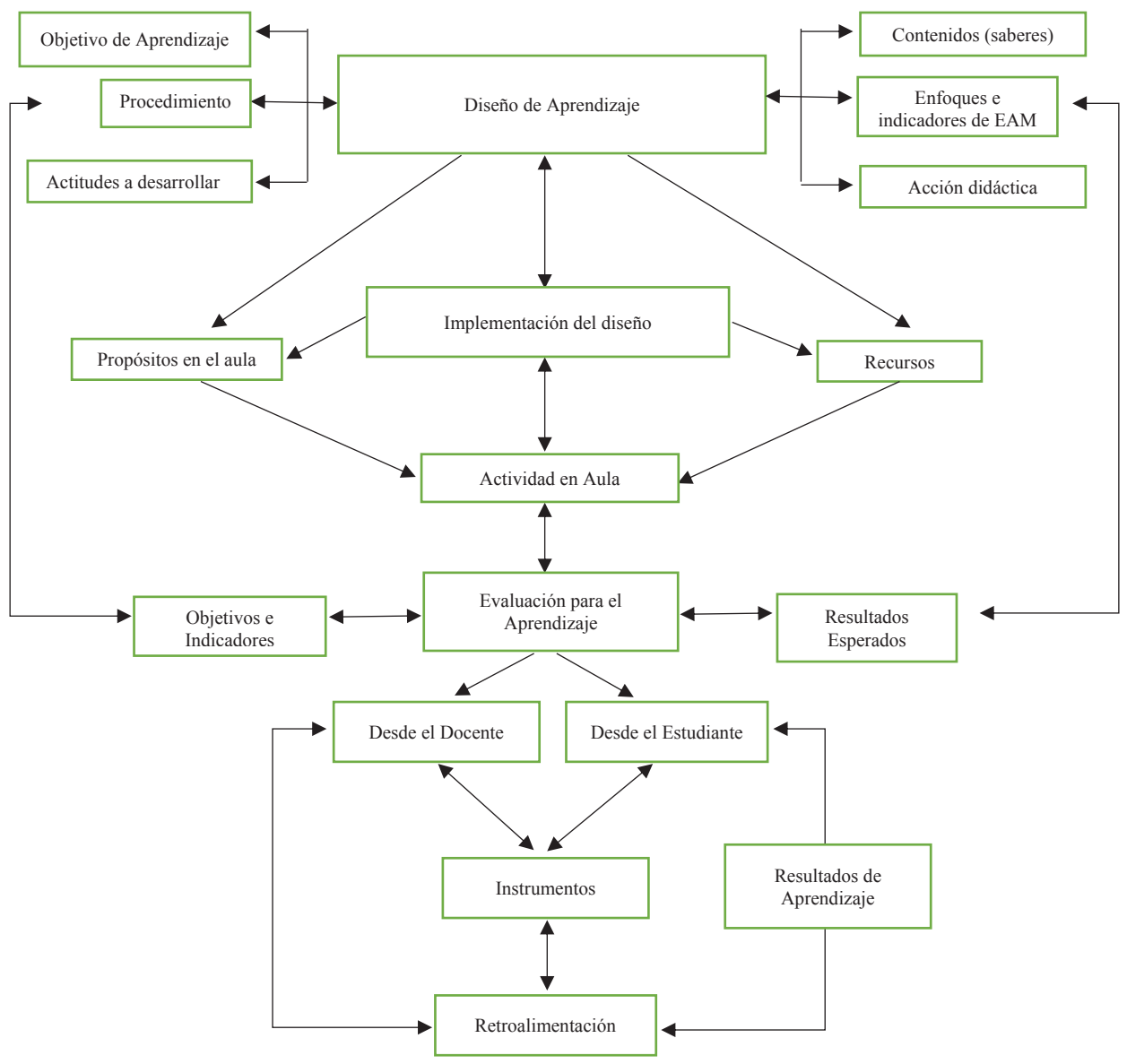

Fuente: elaboración propia.

\section{Diseño del aprendizaje}

El diseño del aprendizaje responde a los lineamientos establecidos por el Ministerio del Medio Ambiente (2009) y al análisis crítico de la EAM que hemos realizado. Aquí se deben integrar y relacionar los factores cognitivos (objetivo de aprendizaje y contenidos), su ejecución — desde indicadores, procedimientos y actitudes—, y, por supuesto, la acción didáctica, la cual debe diseñarse desde el contexto cotidiano de los estudiantes para confluir en una formulación de problema desde una o más preguntas propuestas en la acción pedagógica (Álvarez \& Vega, 2009). Así, los agentes educativos clave son los propios estudiantes que obligan a la adaptación constante del diseño. Por último, la propuesta senda urbana (Soteras \& Naulin, 2013) es una experiencia de estas características en Chile para 
Educación Básica que incorpora los Objetivos de Aprendizaje ministeriales, el rol docente desde una acción didáctica para la EAM, actores asociados al contexto inmediato educativo, como la familia y el barrio, pero también a otros actores tanto públicos como privados.

\section{Implementación en el aula}

Este nivel debe incluir necesariamente la relación entre el Objetivo de Aprendizaje, un diagnóstico de las posibilidades de la escuela o liceo para la implementación de acciones pedagógicas en EAM, propuestas didácticas para el establecimiento de redes cognitivas que propicien la interacción con los estudiantes, el nivel y, si es necesario, lugares o sitios a visitar para complementar la acción. Para esto, proponemos iniciar la secuencia pedagógica desde la formulación propia de un Objetivo de Aprendizaje, que debe responder a la acción evaluativa para la formación de eco-consciencias. Por lo tanto, aquí el "norte" se fundamenta en la praxis desde la perspectiva socio constructivista.

\section{Actividad en el aula}

La determinación aquí es proponer una mirada amplia sobre el aula, donde se incluya espacios educativos como "salidas a terrenos" en el entorno inmediato, a reservas y parques nacionales, pero también al espacio urbano para evidenciar problemáticas y experiencias cotidianas para ser incorporadas a las actividades. Aquí, más que entregar "recetas" de cómo ejecutarlas, nos remitimos a señalar la necesidad de evidenciar una práctica pensada en actitudes proambientales.

\section{Evaluación para el aprendizaje}

La retroalimentación es un factor determinante en esta óptica. Desde el docente y desde el estudiante, sobre todo respondiendo a las interrogantes ¿qué tan significativa fue la retroalimentación?, ¿qué elementos o dimensiones deben ser reforzados o reformulados?, ¿qué hizo o hace con la información de la retroalimentación el estudiante? La retroalimentación como parte fundamental del proceso evaluativo mantiene una relación de dependencia con el proceso de enseñanza y aprendizaje (Chávez \& Larraguibel, 2015). Es una forma de mantener una autorregulación del aprendizaje desde una óptica de mejora constante, en el sentido de la valoración misma de esta construcción del aprendizaje (Coll et al., 2007). Para nuestro objetivo de formación, estas ideas se convierten en ejes primordiales para generar efectos positivos desde la EAM. Tal como es planteado por Coll, Mauri y Rochera (2012) y Nicol (2010), la acción retroalimentadora o feedback del docente debe incluir principios como: ser capaz de orientar al estudiante de manera flexible — no tan general o específico-, dirigir para que capten la idea central, definir principios orientadores, ser operativos para el proceso aprendizaje y un apoyo para la autoevaluación.

Desde el estudiante, la retroalimentación es articulada a partir de los usos, formas y sentidos de esta evaluación con finalidad formadora de eco-consciencias. Además debe incluir las dimensiones de temporalidad — marco para el desarrollo-, finalidad y opciones que permitan el autoaprendizaje. Con estas bases, la idea es convertirla en una instancia formativa encaminada al desarrollo holístico e integral del sujeto desde la integración de una eco-consciencia que impacte su vida cotidiana. Así, la cultura evaluativa y el uso 
del feedback desde esta concepción no solo logra problematizar el aprendizaje, a su vez ofrece oportunidades de mejoramiento continuo en el plano pedagógico con la finalidad de incorporar nuevos enfoques evaluativos que contemplen la retroalimentación como un componente esencial para la interacción entre el proceso de enseñanza-aprendizaje, la actividad de aula y la evaluación. Esta orientación asume la gran posibilidad de convertirla en un método para la formación crítica de eco-conciencias.

\section{CONSIDERACIONES FINALES}

Gran parte de la literatura mencionada plantea el desafío, y al mismo tiempo la ausencia, de la dimensión evaluativa para elaborar diseños educativos y pedagógicos para trabajar activamente la formación de eco-consciencias en el aula.

Para profundizar en el desarrollo de acciones proambientales en el aula y fuera de ella, es necesario incluir en el Proyecto Educativo Institucional (PEI) las dimensiones de la EAM desde una perspectiva integral, para promover desde una racionalidad concreta la formación de sujetos eco-amigables con su entorno. Esto se ha convertido en una necesidad a nivel nacional e internacional: cómo potenciar el desarrollo de ciudadanos responsables con el medio ambiente y comprometidos con el desarrollo sustentable. Como ha sido manifestado en estas páginas, es fundamental integrar nuevas prácticas pedagógicas y evaluativas que respondan a una necesidad que transciende a la educación formal. Pensamos en aprendizajes para toda la vida que cuestionen e influyan conscientemente en la generación de espacios educativos que aporten a la protección de su entorno directo e indirecto con acciones concretas.

La práctica educativa y pedagógica debe estar orientada a la integración y participación de los estudiantes como agentes fundamentales para transformar sus prácticas, tanto al interior como al exterior de la escuela. La idea es ampliar y transferir el fenómeno del aprendizaje medioambiental a los espacios cotidianos de los sujetos como una forma de profundizar y consolidar el desarrollo de una sociedad eco-responsable. Por ello, es urgente incorporar los principios de la EAM de manera transversal en el currículum nacional, convirtiéndolo en un eje primordial en todas las dimensiones del conocimiento. Si bien esta no es la única solución, se deben priorizar también los PEI enfocados con principios reales de acción que promuevan el cuidado del entorno y, sobre todo, que permitan la formación de eco-consciencias para asegurar una mejorar calidad de vida de toda la comunidad. Esto es parte, a nuestro juicio, de una concepción de formación ciudadana mucho más amplia, democrática y participativa, la cual es capaz de interrelacionar la participación política y la acción ciudadana a los ámbitos del consumo responsable, la reutilización, el reciclaje y la protección de los recursos naturales.

Si bien no hemos podido incluir los indicadores y objetivos estratégicos para la formulación de una estructura evaluativa auténtica, en lo sustancial el articular los enfoques de la EAM con los de una cultura evaluativa de carácter socio-crítico y socio constructivista permite visualizar los nuevos desafíos en este frente educativo. Sin embargo, no es posible circunscribir las reflexiones solo para este caso. La evaluación como un componente de mejoramiento continuo orienta una capacidad transformadora al interior del ser, lo que posibilita una formación activa de eco-consciencias en el entendido que la evaluación puede aportar entre otras experiencias escolares a contribuir al desarrollo de eco-aprendizajes en el aula. 
En este sentido, el rol de la retroalimentación — feedback- desde el docente y el estudiante es clave para evidenciar los logros/desempeños o "competencias" adquiridas en torno a la construcción de sujetos responsables con su medio. La intencionalidad formadora que hemos planteado a lo largo del artículo solo puede concretizarse si se logra incorporar espacios de discusión sobre las actividades realizadas en el aula. Tal como ha sido planteado por Coll et al. (2007, 2012), el aprendizaje es una acción transformadora de sentidos del ser. Este resignificar de contenidos abre espacios también para resignificar la acción del sujeto que está en el aula y transferir dicha reflexión individual y colectiva a la conformación de una praxis responsable con su medio ambiente.

Estas reflexiones nos llevan a nuevos caminos e interrogantes sobre la EAM y su dimensión evaluativa: ¿Cuáles serían las mejores estrategias para evidenciar los avances y/o retrocesos en la formación de eco-consciencias?, incluso, ir más allá ¿cómo incorporar en el diseño curricular, desde una mirada transdisciplinaria, la EAM como elemento fundamental para formar a la comunidad y realizar acciones que transciendan meramente al Desarrollo Sostenible?

La educación abre posibilidades que recién están en discusión. Desde nuestro planteamiento hay que ir más allá de la "tradicional" experiencia de EAM, que solo se vincula al "contacto con el medio", a la "salida al entorno natural" o "a elementos pasivos de acción". Se requiere un esfuerzo mayor para generar impactos en los sujetos y así transformar las nociones que tenemos sobre el medio ambiente. Sin embargo, creemos que los pasos "teóricos" de los últimos 20 años han aportado a cambiar la mirada.

Finalmente, esperamos que las discusiones y proposiciones sobre una cultura evaluativa para la formación de eco-consciencias con el Modelo EFEA sea el inicio de nuevas discusiones y aplicaciones reales en el aula. Esta es la deuda: avanzar en orientaciones prácticas desde estas nuevas concepciones.

\section{REFERENCIAS BIBLIOGRÁFICAS}

Ahumada, P. (2001). La evaluación en una concepción de aprendizaje significativo. Valparaíso: Ediciones Universitarias Universidad Católica de Valparaíso.

Ahumada, P. (2005). La evaluación auténtica: un sistema para la obtención de evidencias y vivencias de los aprendizajes. Perspectiva Educacional, 45(1), 11-24.

Álvarez, P., \& Vega, P. (2009). Actitudes ambientales y conductas sostenibles. Implicaciones para la educación ambiental. Revista de Psicodidáctica, 4(2), 245-260.

Cantú, P. C. (2008). Desarrollo sustentable: Conceptos y reflexiones. Nuevo León: Universidad Autónoma de Nuevo León.

Cantú, P. (2014). Educación ambiental y la escuela como espacio educativo para la promoción de la sustentabilidad. Revista Educare, 18(3), 39-52.

Chávez, J., \& Larraguibel, E. (2015). El feedback como herramienta de evaluación formativa y formadora en educación universitaria. Reporte técnico actualmente sometido a evaluación en revista especializada.

Clará, M., \& Mauri, T. (2010). El conocimiento práctico. Cuatro conceptualizaciones constructivistas de las relaciones entre conocimiento teórico y práctica educativa. Infancia y Aprendizaje, 33(2), 131-141.

Coll, C. (2001). Constructivismo y educación: la concepción constructivista de la enseñanza y del aprendizaje. En C. Coll, J. Palacios y A. Marchesi (Comps.), Desarrollo psicológico y educación 
Vol. 2: Psicología de la educación escolar (pp. 157-186). Madrid: Alianza Editorial.

Coll, C., Mauri, T., \& Rochera, M. J. (2012). La práctica de evaluación como contexto para el aprendizaje competente. Revista Profesorado, 16(1), 49-59.

Coll, C., Rochera, M. J., Mayordomo, R. M., \& Naranjo, M. (2007). Evaluación continua y ayuda al aprendizaje. Análisis de una experiencia de innovación en educación superior con apoyo de las TIC. Electronic Journal of Research in Educational Psychology, 5(13), 783-804.

Delors, J. (2006). La educación encierra un tesoro (Compendio). París: UNESCO.

Díaz-Barriga, A. (2014). Construcción de programas de estudio en la perspectiva del enfoque de desarrollo de competencias. Perfiles Educativos, 36(143), 142-162.

Díaz-Barriga, F., \& Barroso, R. (2014). Diseño y validación de una propuesta de evaluación auténtica de competencias en un programa de formación de docentes de educación básica en México. Perspectiva Educacional, 53(1), 36-56.

Edwards, C., Brennan, R., Hardy, I., \& Ponte, P. (2010). Relational architectures: recovering solidarity and agency as living practices in education. Pedagogy, Culture \& Society, 18(1), 43-54.

Escudero, J. (2003). La calidad de la educación: controversias y retos para la Educación Pública. Educatio, 20, 21-38.

Freitas, M. (2006). Educação ambiental e/ou educação para o desenvolvimento sustentável? Uma análise centrada na realidade portuguesa. Revista Iberoamericana de Educación, 41, 133-147.

Gimeno, J. (2001). Educar y convivir en la cultura global. Madrid: Morata.

González Muñoz, M. (1996). Principales tendencias y modelos de la Educación Ambiental en el sistema escolar. Revista Iberoamericana de Educación, 11, 13-74.

Gutiérrez, J., \& Pozo, T. (2006). Modelos teóricos contemporáneos y marcos de fundamentación de la educación ambiental para el desarrollo sostenible. Revista Iberoamericana de Educación, $41(1), 21-68$.

Heikkinen, H. (2011). Understanding induction of new teachers as practice architectures. Symposium in NERA 2011 Supporting Early Career Teachers, 1-12.

Mateo, J., \& Martínez, F. (2008). La evaluación alternativa de los aprendizajes. Barcelona: Octaedro.

Meira, P., \& Caride, J.A. (2006). La geometría de la educación para el desarrollo sostenible, o la imposibilidad de una nueva cultura ambiental. Revista Iberoamericana de Educación, 41, 103116.

Medir, R., Heras, R., \& Magin, C. (2013). Una propuesta evaluativa para actividades de educación ambiental para la sostenibilidad. Educación XXI, 1-25.

Ministerio del Medio Ambiente. (2009). Política Nacional de Educación para el Desarrollo Sustentable. Boletín Oficial.

Ministerio del Medio Ambiente. (2010). Ley 20417. Creación del Ministerio del Medio Ambiente, El Servicio de Evaluación Ambiental y la Superintendencia del Medio Ambiente. Santiago: Biblioteca del Congreso Nacional.

Ministerio del Medio Ambiente. (2015). Primera Encuesta Nacional del Medioambiente. Santiago: Autor.

Ministerio del Medio Ambiente. (2016). Informe Cuenta Pública Participativa Nacional. Santiago: Autor.

Molano, A. (2012). La complejidad de la educación ambiental: una mirada desde los siete saberes para la educación del futuro de Morin. Revista de Didáctica Ambiental, 8(11), 1-9.

Moreno, T. (2010). Lo bueno, lo malo y lo feo: las muchas caras de la evaluación. Revista de Educación Superior, I(2), 84-97.

Nicol, D. (2010). From monologue to dialogue: improving written feedback processes in mass higher education. Assessment y Evaluation in Higher Education, 35(5), 501- 517.

Novo, M. (2006). El desarrollo sostenible. Su dimensión ambiental y educativa. Madrid: Pearson/ UNESCO.

Novo, M. (2009). La educación ambiental, una genuina educación para el desarrollo sostenible. 
Revista de Educación, número extraordinario, 195-217.

Pascual, E. (1998). Racionalidades en la producción curricular y el proyecto curricular. Pensamiento Educativo, 23, 13-72.

Perassi, Z. (2010). ¿En qué medida la evaluación colabora con la mejora escolar? Revista Iberoamericana de Educación, 54(4), 1-18.

Perrenoud, P. (2004). Diez nuevas competencias para enseñar. Barcelona: Graó.

Ravela, P. (2006). Fichas didácticas. Para comprender las evaluaciones educativas. Montevideo: PREAL-Paidós.

Ríos, D. (s/f). Calidad de la educación y su relación con la evaluación de las escuelas. Documento como apoyo a la docencia, Universidad de Santiago de Chile.

Rueda, M. (2004). La evaluación de la relación educativa en la universidad. Revista Electrónica de Investigación Educativa, 6(2), 2-10.

Rueda, M. (2006). Evaluación de la labor docente en el aula universitaria. México: UNAM, Plaza y Valdés.

Ruíz, I., Barraza, L., \& Ceja, M.P. (2009). La educación para la sustentabilidad: análisis y perspectiva a partir de la experiencia de dos sistemas de bachillerato en comunidades rurales mexicanas. Revista El Periplo Sustentable, 16, 139-167.

Salazar, J. (2010). Encuesta de satisfacción estudiantil versus cultura evaluativa de la docencia. Revista Iberoamericana de Educación, 3(1), 120-132.

Sandoval, M. (2012). Comportamiento sustentable y educación ambiental: una visión desde las prácticas culturales. Revista latinoamericana de Psicología, 44(1), 181-196.

Santos Guerra, M. (1993). La evaluación: un proceso de diálogo, comprensión y mejora. Revista Investigación en la Escuela, 20, 23-35.

Sauvé, L. (2000). Para construir un patrimonio de investigación en educación ambiental. Tópicos en Educación Ambiental, 2(5), 51-68.

Sauvé, L. (2001). L'éducation relative à l'environnement: une dimension essentielle de l'éducation fondamentale. In C. Gohier et S. Laurin La formation fondamentale-Un espace à redefinir (pp. 293-318). Montréal: Les Éditions Logiques.

Sauvé, L. (2004). Perspectivas curriculares para la formación de formadores en educación ambiental. Ponencia presentada en el I Foro Nacional sobre la Incorporación de la Perspectiva Ambiental en la Formación Técnica y Profesional. Universidad Autónoma de San Luis de Potosí, México, del 9 al 13 de Junio, 2003. Recuperado de http://www.magrama.gob.es/es/ceneam/articulos-deopinion/2004_11sauve_tcm7-53066.pdf

Sauvé, L. (2005). Una cartografía de educación ambiental. En M. Sato y C. I. Moura-Carvalho (Eds.), Educação ambiental (pp. 17-44). Sao Paulo: Artmed.

Soteras, C., \& Naulin, P. (2013). Diseño de sendas urbanas en los sectores aledaños a las escuelas. En Ministerio del Medio Ambiente (Ed.), Educación Ambiental: Experiencias metodológicas (pp. 103-149), Santiago: Maval.

Stubbs, W., \& Schapper, J. (2011). Two approaches to curriculum development for educating for sustainability and CSR. International Journal of Sustainability in Higher Education, 12(3), 259268.

UNESCO. (1972). Convención sobre la protección del patrimonio mundial, cultural y natural. Conferencia General de la Organización de las Naciones Unidas para la Educación, la Ciencia y la Cultura. París: Autor.

UNESCO. (2000). Marco de Acción de Dakar. Educación para Todos: cumplir nuestros compromisos comunes. Foro Mundial sobre la Educación. Dakar: Autor.

UNESCO. (2005). Educación para Todos. El Imperativo de la Calidad. París: Autor.

Vallejo, M., \& Molina, J. (2014). La evaluación auténtica de los procesos educativos. Revista Iberoamericana de Educación, 64, 11-25.

Vega, P., \& Álvarez, P. (2005). Planteamiento de un marco teórico de la Educación Ambiental para un 
desarrollo sostenible. Revista Electrónica de Enseñanza de las Ciencias, 4(1), 1-17.

Vega, P., Freitas, M., Álvarez, P., \& Fleuri, R. (2007). Marco teórico y metodológico de educación ambiental e intercultural para un desarrollo sostenible. Revista Eureka, 4(3), 539-554.

Velásquez, J. (2009). La transversalidad como posibilidad curricular desde la educación ambiental. Revista Latinoamericana de Estudios Educativos, 5(2), 29-44.

Vilches, A., \& Gil, D. (2015). Ciencia de la Sostenibilidad: ¿una nueva disciplina o un nuevo enfoque para todas las disciplinas? Revista Iberoamericana Educación, 69(1), 39-60.

Zambrano, J.G., Castillo, M., \& Sánchez, A. (2011). El desarrollo sustentable y los programas educativos ambientales. CONHISREMI, Revista interuniversitaria arbitrada de investigación y diálogo académico, 7(1), 68-85. 
